\title{
TRPV4 channels regulate tumor angiogenesis via modulation of Rho/Rho kinase pathway
}

\author{
Roslin J. Thoppil ${ }^{1,2, *}$, Holly C. Cappelli ${ }^{1,2, *}$, Ravi K. Adapala ${ }^{1,2}$, Anantha K. Kanugula ${ }^{1}$, \\ Sailaja Paruchuri ${ }^{3}$, Charles K. Thodeti, ${ }^{1,2}$ \\ ${ }^{1}$ Department of Integrative Medical Sciences, Northeast Ohio Medical University, $\mathrm{OH} 44272$, Rootstown, USA \\ ${ }^{2}$ School of Biomedical Sciences, Kent State University, OH 44240, Kent, USA \\ ${ }^{3}$ Department of Chemistry, University of Akron, $\mathrm{OH} 44325$, Akron, USA \\ *These authors contributed equally to this work
}

Correspondence to: Charles K. Thodeti, e-mail: cthodeti@neomed.edu

Keywords: endothelial cell, mechanotransduction, Rho/Rho kinase, TRPV4, tumor angiogenesis

Received: January 29, $2016 \quad$ Accepted: March 10, $2016 \quad$ Published: March 26, 2016

\section{ABSTRACT}

Targeting angiogenesis is considered a promising therapy for cancer. Besides curtailing soluble factor mediated tumor angiogenesis, understanding the unexplored regulation of angiogenesis by mechanical cues may lead to the identification of novel therapeutic targets. We have recently shown that expression and activity of mechanosensitive ion channel transient receptor potential vanilloid 4 (TRPV4) is suppressed in tumor endothelial cells and restoring TRPV4 expression or activation induces vascular normalization and improves cancer therapy. However, the molecular mechanism(s) by which TRPV4 modulates angiogenesis are still in their infancy. To explore how TRPV4 regulates angiogenesis, we have employed TRPV4 null endothelial cells (TRPV4KO EC) and TRPV4KO mice. We found that absence of TRPV4 (TRPV4KO EC) resulted in a significant increase in proliferation, migration, and abnormal tube formation in vitro when compared to WT EC. Concomitantly, sprouting angiogenesis ex vivo and vascular growth in vivo was enhanced in TRPV4KO mice. Mechanistically, we observed that loss of TRPV4 leads to a significant increase in basal Rho activity in TRPV4KO EC that corresponded to their aberrant mechanosensitivity on varying stiffness ECM gels. Importantly, pharmacological inhibition of the Rho/Rho kinase pathway by Y-27632 normalized abnormal mechanosensitivity and angiogenesis exhibited by TRPV4KO EC in vitro. Finally, Y-27632 treatment increased pericyte coverage and in conjunction with Cisplatin, significantly reduced tumor growth in TRPV4KO mice. Taken together, these data suggest that TRPV4 regulates angiogenesis endogenously via modulation of EC mechanosensitivity through the Rho/Rho kinase pathway and can serve as a potential therapeutic target for cancer therapy.

\section{INTRODUCTION}

A key hallmark in the growth and progression of solid tumors is the sustained stimulation of angiogenesis [1]. Comprised of complex and tightly regulated processes, including basement membrane remodeling, endothelial cell (EC) proliferation, migration, and tube formation, angiogenesis plays a critical role in cancer. From the beginning of tumorigenesis, tumor cells cultivate an environment with an abundance of pro-angiogenic factors to allow for the formation of blood vessels that can infiltrate the solid tumor [2-3]. Tumor angiogenesis is an important control point for the progression of any solid tumor however, the resulting vasculature is highly abnormal, making the delivery of anti-cancer therapies difficult [4]. These challenges led to the development of anti-angiogenic therapies, which aim to target tumor angiogenesis as opposed to solely suppressing tumor growth. Many of these studies have focused on pro-angiogenic signaling pathways but have been met with limited success due to acquired resistance and/or impaired drug delivery, as well as their adverse effects on normal tissue [5-6]. Therefore, the identification of alternative therapies that target tumor angiogenesis are still of high value. 
Emerging evidence has shown that the transient receptor potential (TRP) superfamily of ion channels is associated with a variety of cancers [7]. Aberrant functions of these channels can lead to sustained proliferative signaling, evading growth suppressors, resisting cell death, and increased production and secretion of mitogens, which are closely related with the defined hallmarks of cancer [8-11]. Further, many of these cell processes are highly sensitive to changes in $\left[\mathrm{Ca}^{2+}\right]_{\mathrm{i}}$ which makes TRP channels an attractive avenue to explore. TRPV4 channels play an important role in regulating EC physiology via mechanotransduction [12-19]. Our latest findings revealed that decreased functional expression of TRPV4 plays a role in the aberrant mechanical properties of TEC (tumor endothelial cells), which we were able to rescue with overexpression or pharmacological activation of TRPV4. We further demonstrated that TRPV4 activation normalized the tumor vasculature in WT mice injected with tumors, to promote efficient chemotherapeutic drug delivery and reduce overall tumor growth [12]. Taken together, these findings provide compelling evidence that TRPV4 influences angiogenesis however, the molecular mechanism by which TRPV4 regulates angiogenesis remains unknown.

In recent years, the Rho/Rho kinase pathway has become an attractive target in cancer medicine due to its involvement in cellular processes such as proliferation, cell shape, motility, as well as its contribution to tumor progression [20]. Several studies have shown that inhibition of the Rho/Rho kinase signaling pathway can decrease tumor cell proliferation and invasion in vitro as well as decrease tumor growth and metastasis in vivo [21-26]. Further, the Rho/Rho kinase pathway has been found to be involved in EC permeability, migration, and survival and has been found to play an integral part in vascular endothelial growth factor (VEGF)-mediated angiogenesis [27-28]. Although Rho/Rho kinase inhibitors have been used in a variety of cancer models to study tumor progression and/or metastasis [23, 29, 30], neither their effects on tumor angiogenesis and vascular normalization nor upstream regulators of this pathway have been identified. In the present study, we explored the molecular mechanism by which TRPV4 regulates angiogenesis by focusing on the Rho/Rho kinase pathway.

\section{RESULTS}

\section{TRPV 4 deletion induces increased EC proliferation, migration, and basal Rho activity}

To determine the molecular mechanism by which TRPV4 may influence angiogenesis, we first isolated EC from the vascular sprouts that originated from WT and TRPV4KO aortic explants. EC were cultured in defined media and characterized by measuring the expression of endothelial cell markers, smooth muscle cell markers, and TRPV4, using immunofluorescence, Western blot, and qPCR analysis (Supplementary Figures S1 and S2). EC from both WT and TRPV4KO explants exhibited an endothelial phenotype in culture (Supplementary Figure S1A), as evidenced by the expression of EC marker CD31 (Supplementary Figure S1B) and absence of smooth muscle cell marker, alpha-SMA (Supplementary Figure S1C), confirming that these are bonafide EC. Western blot analysis revealed TRPV4 specific bands (two bands, one below and above $100 \mathrm{kDa}$ ) in WT EC, which were absent in TRPV4KO EC (Supplementary Figure S2A, S2B).

EC proliferation and migration are important events in angiogenesis, which rely on the mechanosensing ability of EC to coordinate cytoskeletal reorganization and changes in cell adhesion [31-32]. Therefore, we hypothesized that lack of the mechanosensor TRPV4 would alter EC proliferation and migration. Indeed, we found that TRPV4KO EC showed increased proliferation (Figure 1A). We also found that this increased proliferation correlated with enhanced ERK1/2 phosphorylation in TRPV4KO EC (Figure 1B). Further, we found that TRPV4KO EC migrated significantly $(p \leq 0.001)$ greater than normal EC (NEC) (Figure 1C). These findings are reminiscent to the increased proliferation and migration we observed in tumor-derived EC (TEC) [12]. Furthermore, we have previously shown that high basal Rho activity, which controls the actin cytoskeleton, contributes to the abnormal mechanosensing ability of TEC, and that TRPV4 activation and/or overexpression can restore Rho activity to that comparable to NEC [12], suggesting that TRPV4 may regulate this pathway. Therefore, to explore whether the complete deletion of TRPV4 modulates EC migration via the Rho pathway, we performed Rho activity assays in WT EC and TRPV4KO EC. As hypothesized, we found that TRPV4KO EC exhibited significantly increased basal Rho activation (Figure 1D). Taken together, these results suggest that TRPV4 is required for the regulation of $\mathrm{EC}$ function by mediating optimal levels of Rho.

\section{Absence of TRPV4 induces abnormal angiogenesis in vitro, ex vivo, and in vivo}

To investigate whether the inherent abnormalities associated with TRPV4KO EC (enhanced proliferation and migration) may also be translated in the way these cells form tubes, we performed 2D angiogenesis assays. We found that when plated on Matrigel, WT EC formed tubular structures within $4 \mathrm{~h}$, which were stable until $8 \mathrm{~h}$. In contrast, TRPV4KO EC formed tubes within $4 \mathrm{~h}$, but then underwent multicellular retraction and tubular disruption to collapse after only $6 \mathrm{~h}$ (Figure 2A), suggesting abnormal angiogenesis by these cells. 
To explore if TRPV4 expression also regulates angiogenesis, we examined vascular sprouting by performing ex vivo aortic ring assays. Using aortas isolated from WT and TRPV4KO mice, we incubated the aortic rings in Matrigel and monitored the growth of vascular sprouts for several days. We found that the aortic rings from TRPV4KO mice produced more sprouts and quantification of sprouts after 5 days of incubation showed significantly enhanced sprout formation from TRPV4KO compared to WT (Figure 2B). To further confirm these findings, we used in vivo Matrigel plug

A

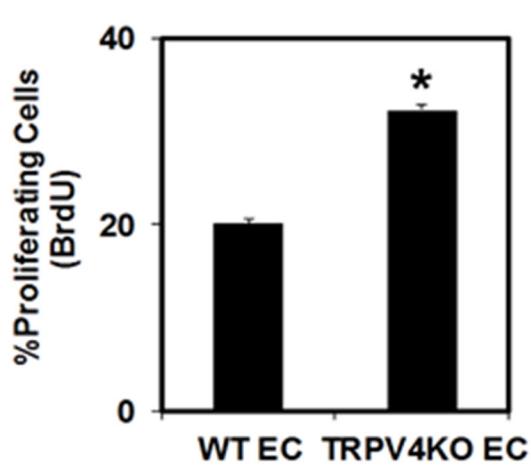

C

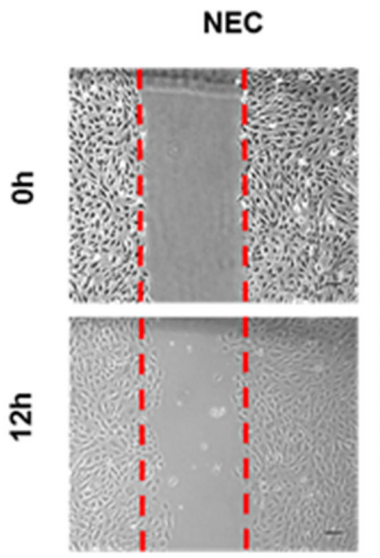

TRPV4KO EC

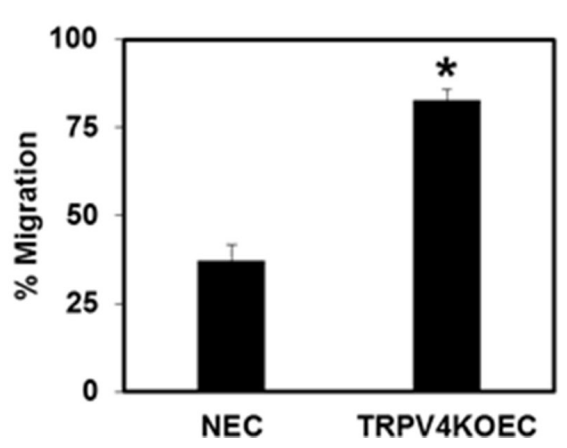

assays. Matrigel plugs supplemented with VEGF and FGF were subcutaneously injected into WT and TRPV4KO mice. Two weeks after the initial injection, Matrigel plugs were excised and the newly invaded endothelial cells were assessed for vessel formation by CD31 and DAPI staining (Figure 2C). Quantitative analysis of DAPI revealed an increase in the number of invaded cells, suggesting that EC may proliferate more in the TRPV4KO mice compared to WT (Supplementary Figure S3). Taken together, these data confirm that the absence of TRPV4 enhances angiogenesis in vitro, ex vivo, and in vivo.

B

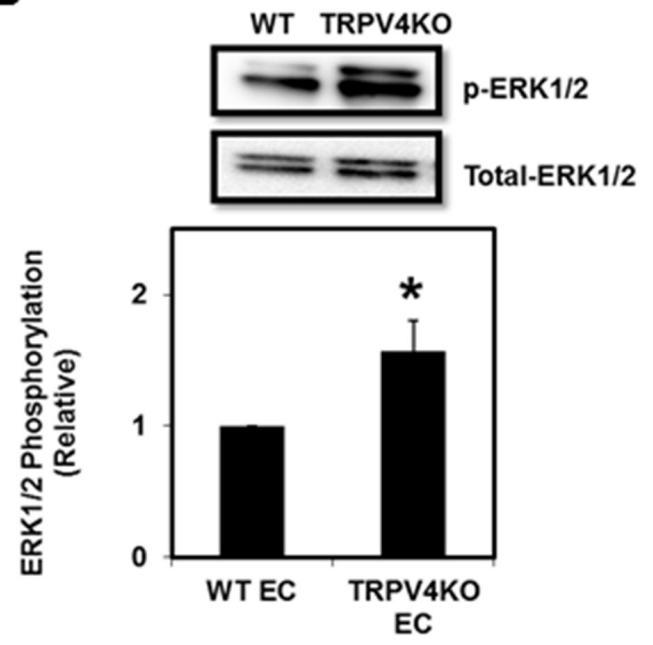

D

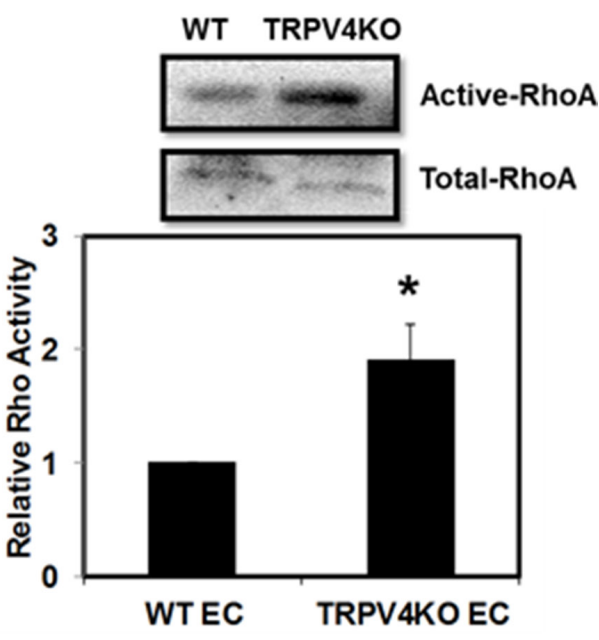

Figure 1: TRPV4 deletion induces abnormal EC proliferation, ERK1/2 phosphorylation, migration, and Rho activity. (A) Quantitative analysis showing increased percentage of BrdU incorporation in TRPV4KO EC compared to WT EC. (B) Above: Western blot showing increased basal phosphorylation of ERK1/2 in TRPV4KO EC. Below: Densitometric analysis of ERK1/2 activity (normalized to total ERK1/2) showed a significant increase in TRPV4KO EC. (C) Above: Representative Brightfield images $(4 \times)$ of scratch wounds taken at 0 and $12 \mathrm{hr}$. Scale bar $=100 \mu \mathrm{m}$. Below: Quantitative analysis showing increased migration of TRPV4KO EC compared to normal EC (NEC). (D) Above: Western blot showing increased basal levels of active RhoA in TRPV4KO EC compared to WT EC. Below: Densitometric analysis of RhoA activity (normalized to total RhoA) showed a significant increase in TRPV4KO EC. 


\section{Pharmacological inhibition of the Rho/Rho} kinase pathway restores mechanosensitivity and normalizes angiogenesis in TRPV4KO EC

The above findings suggest that in the absence of TRPV4, increased Rho/Rho kinase signaling may contribute to the aberrant mechanosensitivity of EC to ECM stiffness. To explore if the Rho/Rho kinase pathway influences TRPV4KO EC mechanosensitivity towards ECM stiffness, we next cultured NEC and TRPV4KO EC on transglutaminase linked gelatin gels of varying stiffness
(98, 370, and $2280 \mathrm{~Pa}$; representing low, intermediate, and high stiffness, respectively) [12,33], which mimic the stiffness of tumor ECM [34-35]. Cells were seeded equally and allowed to spread for $6 \mathrm{~h}$ in the presence and absence of Y-27632 $(10 \mu \mathrm{M})$. When we compared the degree of spreading of NEC, we found that the cell area increased from low to intermediate stiffness, and reached a plateau at the highest stiffness (Figure 3A), which was similar to our previous study [33]. On the other hand, while the TRPV4KO EC followed a similar trend, the spreading on intermediate and high stiffness gels
A
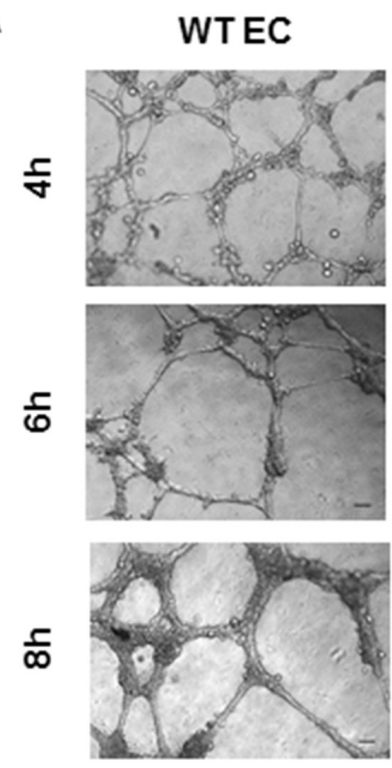

C
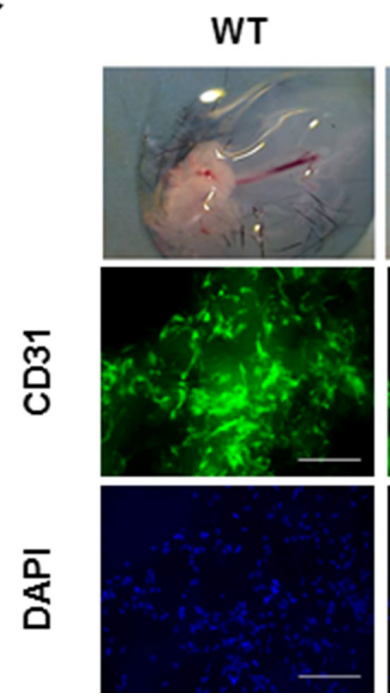

TRPV4KOEC
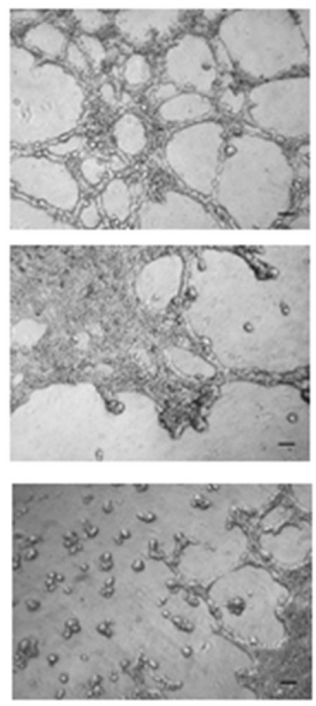

TRPV4KO
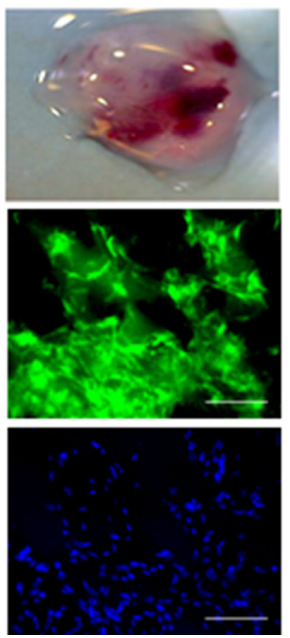

B
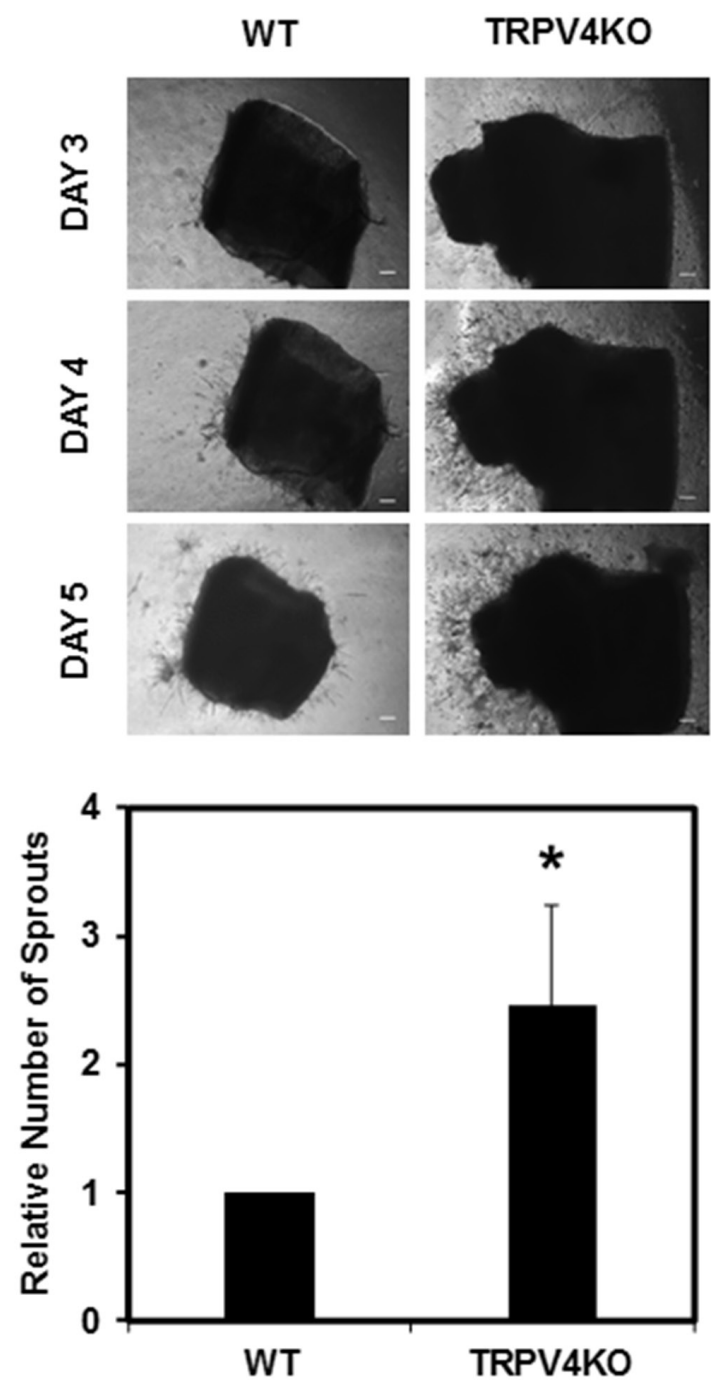

Figure 2: Absence of TRPV4 induces abnormal angiogenesis in vitro, ex vivo, and in vivo. (A) Phase contrast images (4×) showing the angiogenic behavior of WT EC and TRPV4KO EC when plated on 2D Matrigel at high density $\left(8 \times 10^{4} /\right.$ well) at 4, 6, and 8 hours. (B) Above: Representative images $(4 \times)$ showing sprouting angiogenesis from aortic explants isolated from WT and TRPV4KO mice. Below: Quantitative analysis showing significantly $(p \leq 0.05)$ increased vascular sprouting in aortic explants from TRPV4KO mice on Day 5. (C) Images of Matrigel plugs extracted from WT and TRPV4KO mice. Immunofluorescence images from Matrigel plugs sections (WT and TRPV4KO) showing vessel formation, as visualized by CD31 staining. Green = CD31; Blue = DAPI staining for nuclei. Scale bar $=100 \mu \mathrm{m}$ for all images. 
was significantly more than at low stiffness (Figure 3A). Notably, treatment with Y-27632 reduced the cell spreading of TRPV4KO EC on both intermediate and high stiffness gels compared to untreated cells (Figure 3A), suggesting that pharmacological inhibition of Rho kinase with Y-27632 restored substrate mechanosensitivity in these cells. In contrast, Y-27632 had no significant effect on NEC spreading at low, intermediate, or high stiffness substrates. These results suggest that inhibition of the Rho/Rho kinase pathway restores mechanosensitivity in TRPV4KO EC.

Next, we asked if normalizing Rho or Rho kinase (ROCK; downstream effector of Rho) activity would normalize tube formation in TRPV4KO EC.
To achieve this, we performed 2D angiogenesis experiments in the presence of Rho kinase inhibitor Y-27632 $(10 \mu \mathrm{M})$ (Figure 3B). We found that in the absence of Y-27632, TRPV4KO EC formed tubes at earlier time points but collapsed after $6 \mathrm{~h}$. However, these cells formed robust tubes in the presence of Y-27632, which were comparable to WT EC, stable after $8 \mathrm{~h}$, and resulted in a significant increase in tube length (Figure 3B). Further, Y-27632 treatment had no significant effect on WT EC tube formation (data not shown). These results suggest that TRPV4 regulates angiogenesis via modulation of Rho signaling, and that the absence of TRPV4 results in abnormal angiogenesis.
A
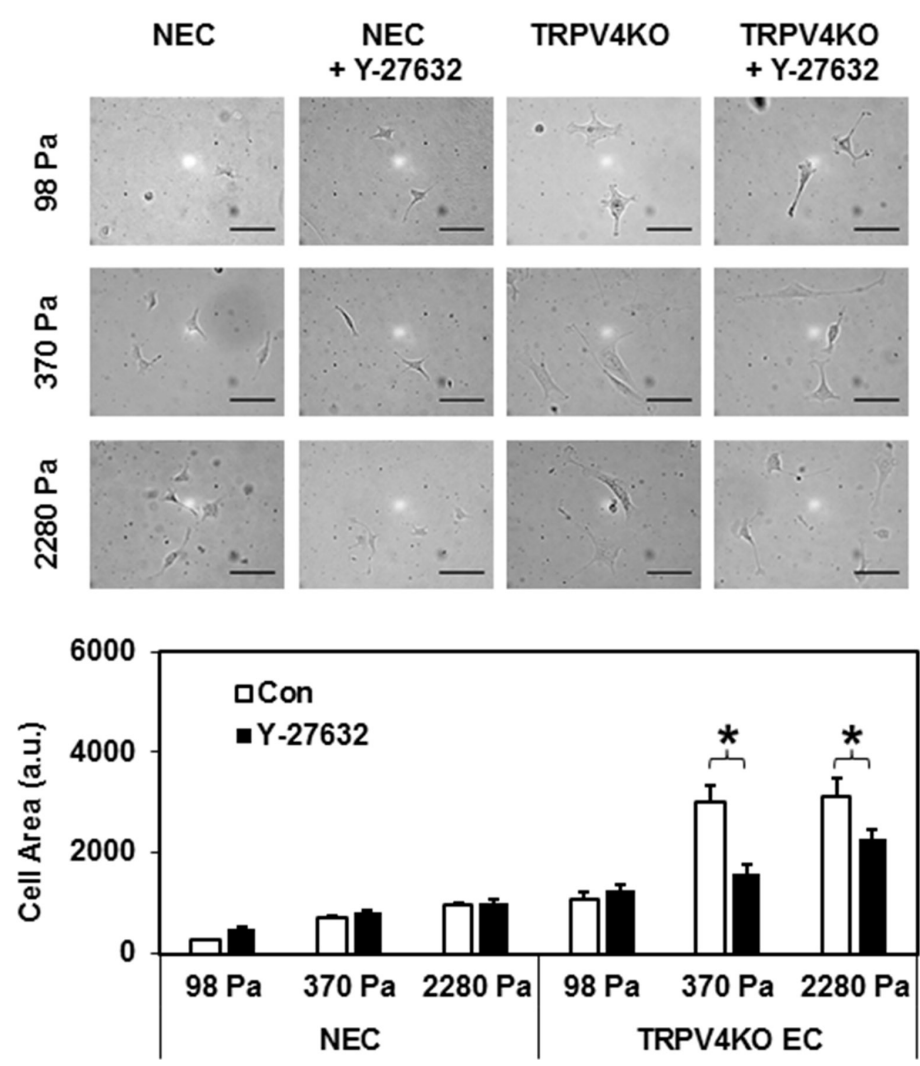

B
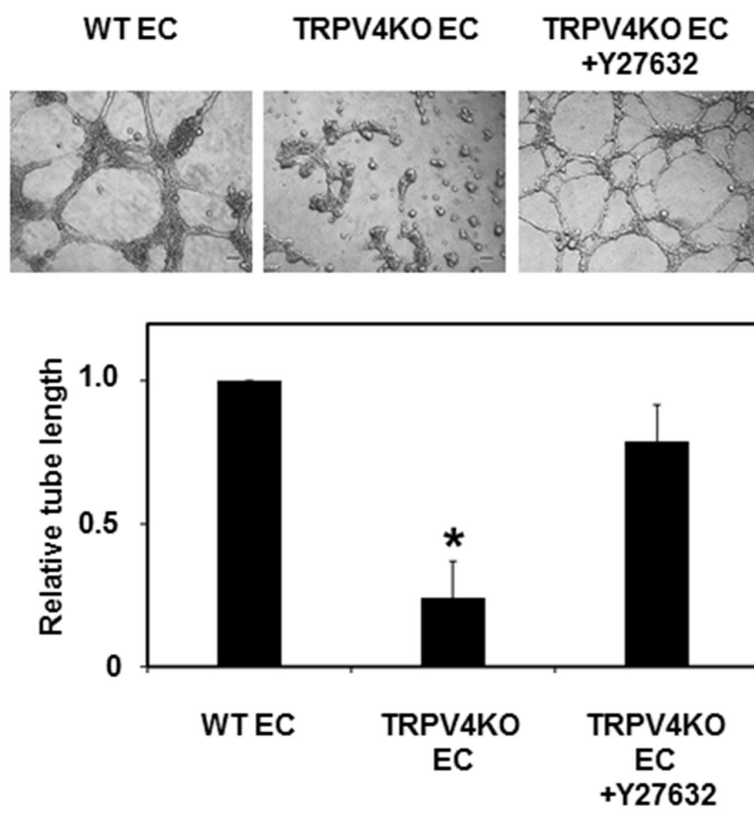

Figure 3: Rho kinase (ROCK) inhibition normalizes abnormal mechanosensitivity and angiogenesis exhibited by TRPV4KO EC. (A) Above: Phase contrast micrographs (20X) showing the normalizing effects of Rho kinase inhibitor, Y-27632 $(10 \mu \mathrm{M})$ on TRPV4KO EC spreading. Cells were equally plated on ECM gels of varying stiffness $(98,370$, and $2288 \mathrm{~Pa})$ and representative images were taken $6 \mathrm{~h}$ after plating. Scale bar $=100 \mu \mathrm{m}$. Below: Quantification of cell area revealed that while treatment with Y-27632 $(10 \mu \mathrm{M})$ had no effect on NEC spreading, Y-27632 significantly attenuated abnormal TRPV4KO EC spreading at intermediate and high stiffness gels. (B) Above: Phase contrast micrographs (4×) showing the normalizing effects of Rho kinase inhibitor, Y-27632 (10 $\mu \mathrm{M})$ on TRPV4KO EC angiogenic behavior, when plated on Matrigel at high density $\left(8 \times 10^{4}\right.$ well). WT EC were used as a control. Representative images were taken $8 \mathrm{~h}$ post plating, showing the formation of stable tubes upon inhibition of the Rho kinase pathway. Scale bar $=100 \mu \mathrm{m}$. Below: Quantification of tube length revealed a significant increase $(p \leq 0.05)$ in tube formation in TRPV4KO EC+Y-27632 compared to untreated TRPV4KO EC controls. Further, the measured tube lengths of TRPV4KO EC treated with Y-27632 were comparable to WT EC. 


\section{Rho kinase inhibitor Y-27632, in conjunction with Cisplatin, reduces tumor growth in TRPV4KO mice}

Our results clearly demonstrate that deletion of TRPV4 (TRPV4KO EC) results in increased Rho activation and angiogenesis in vitro, ex vivo, and in vivo. Further, our in vitro results showed that inhibition of the Rho pathway with Y-27632 normalized abnormal EC function and angiogenesis. Based on these findings, we hypothesized that Y-27632 treatment may reduce tumor growth in TRPV4KO mice. To accomplish this, we injected Lewis Lung Carcinoma (LLC) cells into TRPV4KO mice. Once the tumors became palpable (after $\sim 7$ days), we injected Y-27632 (i.p., $10 \mathrm{mg} / \mathrm{kg}$ ) every day for 14 days. Saline injected mice served as controls. Additionally, to examine if Rho kinase inhibition normalizes tumor vasculature and improves cancer therapy, we treated mice with anti-cancer drug, Cisplatin (i.p., $3 \mathrm{mg} / \mathrm{kg}$ ), once per week, alone and in combination with Y-27632. When used in combination with Y-27632, Cisplatin was administered 2-4 days after Y-27632 treatment. Overall, the TRPV4KO mice were divided into four groups: 1) Control 2) Y-27632 3) Cisplatin and 4) Y-27632 + Cisplatin. Tumor growth and angiogenesis was monitored as previously described [12]. We found that time dependent tumor growth in the control animals reached around $2000 \mathrm{~mm}^{3}$ at 21 days, consistent with our previous findings in TRPV4KO mice [12]. Interestingly, treatment with Y-27632 or Cisplatin alone did not have an effect on overall tumor size, but when used in combination, we found a significant reduction in tumor growth (Figure 4A). These results also suggest that Rho kinase inhibition may have normalized the tumor vasculature, which aided in the efficient and systemic delivery of Cisplatin. Consistent with this observation, we found increased pericyte coverage in tumor vessels that were treated with Y-27632 (Y-27632 and Y-27632 + Cisplatin), but not in the control or Cisplatin alone treated mice (Figure 4B and 4C). Together, our data provides compelling evidence that the Rho/Rho kinase pathway acts downstream of TRPV4 and that TRPV4 modulates angiogenesis by maintaining optimal levels of Rho/Rho kinase activity.

\section{DISCUSSION}

Current and emerging anti-angiogenic therapies are still focused on growth factor signaling pathways, and while there has been therapeutic effects seen in mouse models and some human cancers, the clinical benefits are transitory at best, due to the inevitable development of resistance to the drug [36-37]. Therefore, the need to find alternative therapeutic approaches still remains. Calcium plays a multi-faceted role within the cell, and its involvement in regulating angiogenic processes makes it an important contributor, as well as potential molecular target, for tumor neovascularization and progression [38-40]. Recent reports suggest that transient receptor potential (TRP) channels, many of which are calcium permeable, play a crucial role in the biology of the tumor [41] and their expression in vascular endothelial cells may contribute in regulating tumor angiogenesis. In fact, it has been implicated that members from the TRPC and TRPV subfamilies are involved in endothelial function. TRPC1 and TRPC6 have been found to mediate pro-angiogenic effects by playing a role in VEGF-induced calcium influx [42-44]. More specifically, TRPC6 expression in HUVECs is critical for VEGF-induced proliferation and tube formation in vitro [45]. Moreover, TRPV1 has been found to play a pro-angiogenic role in vivo, where $\mathrm{Yu}$ et al. found that TRPV1 knockdown in zebrafish resulted in severe angiogenic defects [44]. While these channels have been identified to play a role in endothelial function and angiogenesis, little to no studies have implicated a specific role for these channels in the tumor vasculature.

TRPV4 is a ubiquitously expressed mechanosensor of shear stress and cyclic strain in the vascular endothelium [15, 16, 18, 19, 46]. Kohler and colleagues have demonstrated that TRPV4 is critical for sensing shear stress and endothelium-dependent vasodilation $[14,15,47]$. We have also shown that TRPV4 mediates cyclic strain-induced EC reorientation via integrin to integrin signaling [19]. Further, we found that EC derived from the tumor (TEC) fail to reorient in response to cyclic strain and exhibit abnormal angiogenesis in vitro [33]. Recently, we found low functional levels of TRPV4 in TEC, which may contribute to constitutively active Rho, abnormal mechanosensing, and tumor angiogenesis exhibited by these cells. Indeed, our latest studies demonstrated that directly targeting TRPV4 through pharmacological activation normalizes tumor angiogenesis and improves cancer therapy in vivo [12]. However, the precise mechanism by which TRPV4 may regulate angiogenesis remains to be elucidated.

In this study, we provided evidence that absence of mechanosensitive ion channel, TRPV4, acts as a negative regulator of angiogenesis. Recent studies have shown that TRPV4 deficiency leads to increased proliferation of different cell types, including renal duct cells and cholangiocytes [48, 49], and our previous study demonstrated that TRPV4-deficient TEC exhibit increased proliferation and migration $[12,50]$. Therefore, we sought to explore how the complete absence of TRPV4 affected these processes. Here, our results show EC isolated from TRPV4KO mice clearly displayed increased proliferation and migration as well as abnormal tube formation. Further our ex vivo and in vivo results showed an increase in vascular sprouting from aortic rings as well as vascular growth in Matrigel plugs that 
were isolated from TRPV4KO mice, supporting the idea that TRPV4 negatively regulates EC function during angiogenesis

The Rho/Rho kinase pathway also plays an essential role in EC function and angiogenesis by regulating focal adhesion and stress fiber formation during cell migration [51-54]. Further, the tumor microenvironment contains vasculature surrounded by stiff tissue and ECM resulting in increased Rho/Rho kinase signaling in order to balance the external force with the internal cytoskeletal structure [55]. As a result, recent studies have begun investigate this pathway as a potential strategy to prevent tumor progression [28, 56-58] and inhibition of Rho kinase has started to show promise as a vascular normalizing agent. Therefore, to determine if the Rho/Rho kinase pathway is modulated by TRPV4-dependent mechanotransduction, we measured Rho activity and found that the absence of TRPV4 significantly increases basal Rho, suggesting that TRPV4 may regulates tumor angiogenesis by maintaining optimal levels of Rho.
Therefore, we next explored tube formation using 2D angiogenesis assays. When comparing WT EC and TRPV4KO EC, we found that TRPV4KO EC failed to stabilize and collapsed, which corresponds to high basal Rho activity. When we treated the cells with Rho/ Rho kinase pathway inhibitor, Y-27632, we were able to normalize the abnormal angiogenesis displayed by TRPV4KO EC. Next, we asked if the Rho/Rho kinase pathway is the underlying molecular mechanism for enhanced tumor growth and tumor angiogenesis in TRPV4KO mice. We found that inhibition of the Rho/ Rho kinase pathway in vivo in TRPV4KO mice treated with Y-27632 was able to normalize tumor vasculature, as evidenced by increased pericyte coverage of tumor vessels. This vascular normalization by Y-27632 in TRPV4KO tumors was further confirmed by reduced tumor growth when treated simultaneously with Y-27632 and Cisplatin.

Recent work has started to highlight the role of mechanical forces in angiogenesis, as mechanical forces have been shown to modulate EC responses to
A

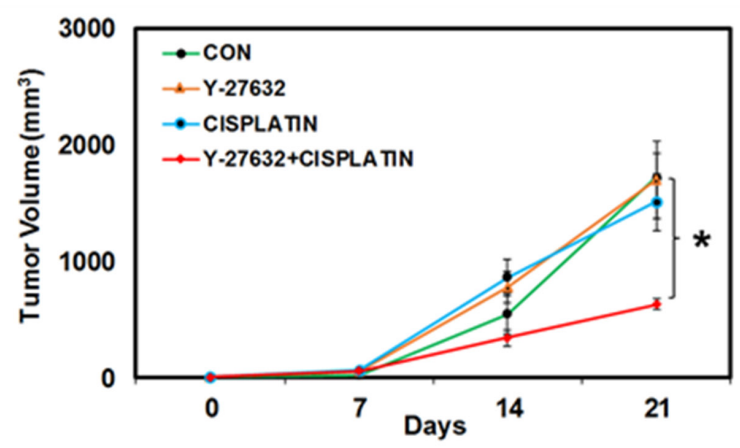

B

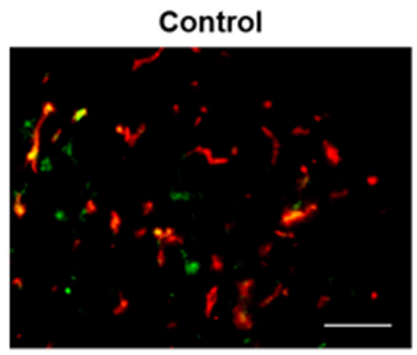

C

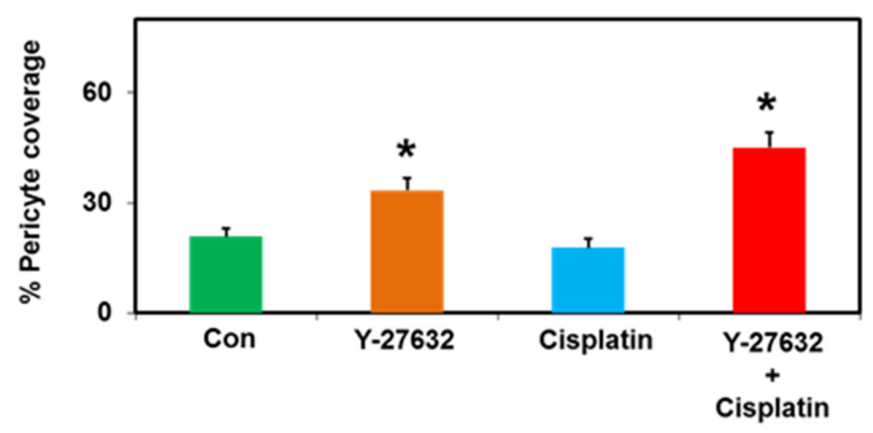

Cisplatin
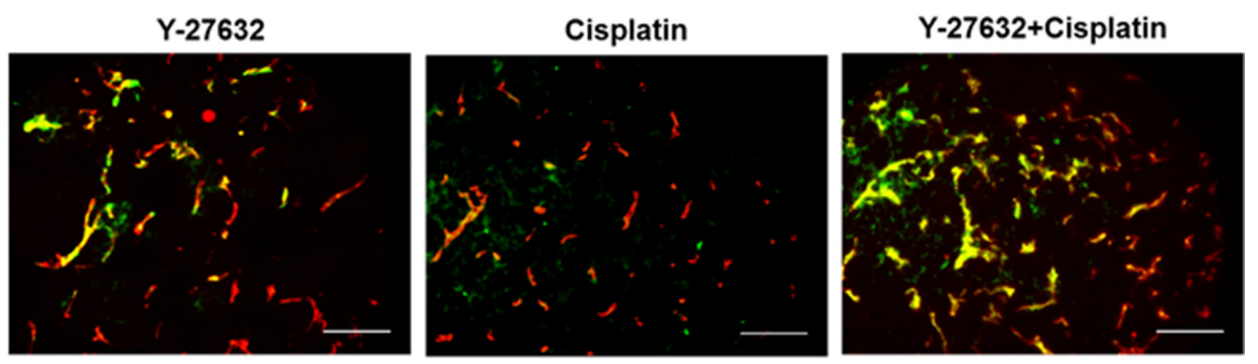

CD31/ $\alpha-S M A$

Figure 4: ROCK inhibitor, Y-27632, in conjunction with Cisplatin, reduces tumor growth in vivo in TRPV4KO mice. (A) Time-dependent growth of tumors in mice injected with saline (CON), Y-27632, Cisplatin, or Y-27632 + Cisplatin. Syngeneic tumors (LLC) were injected in the back of TRPV4KO mice and tumor growth was monitored for 21 days. Rho kinase inhibitor, Y-27632, was injected (i.p.) $(10 \mathrm{mg} / \mathrm{kg})$ every day starting from Day 7 until Day 21. Cisplatin was injected (i.p.) (3 mg/kg) once per week starting 2-4 days after the injection of Y-27632. Note that tumor growth was significantly reduced in Y-27632+Cisplatin treated mice, but not Y-27632 or Cisplatin alone. (B) Frozen sections of tumors from Control, Y-27632, Cisplatin, and Y-27632+Cisplatin treated mice (10 $\mu \mathrm{m}$ thickness; from Day 21) were stained with CD31 (red) and $\alpha$-SMA (green) to measure pericyte coverage (matured vessels). Scale bar $=100 \mu \mathrm{m}$. (C) Quantitative analysis of pericyte covered micro-vessels showing increased pericyte coverage in the tumor vessels treated with Y-27632 or Y-27632 + Cisplatin, but not in Cisplatin alone treated mice. 
growth factors. Importantly, changes in the balance between contractile (mechanical) forces generated by the cytoskeleton and the ECM regulate cell shape, proliferation, migration, and ultimately survival. In fact, cellular tension is sustained and regulated, to a large extent, by the Rho/Rho kinase pathway [31, 32, 59-61]. Although the role of the Rho/Rho kinase pathway has been studied extensively in response to growth factors such as VEGF [28, 62], upstream mechanotransduction pathways that regulate Rho/Rho kinase are not known. Our present study revealed that TRPV4, a mechanosensor in $\mathrm{EC}$, is an endogenous upstream regulator of Rho/Rho kinase-dependent mechanotransduction and angiogenesis
(Figure 5). Although anti-VEGF strategies have been suggested to be vascular normalizing agents, their success in clinics has been very limited. Importantly, recent PET imaging clearly demonstrated that anti-VEGF treatment rapidly (within 4 hours) attenuated perfusion to the tumors, which may act as a barrier to chemo and radiation therapy [63]. On the other hand, most of the work on the Rho/Rho kinase pathway is focused on targeting tumor cell proliferation and migration with little focus on tumor vasculature. Thus, our current findings propose that TRPV4 can be used as an alternate therapeutic target to inhibit/normalize tumor angiogenesis via modulation of Rho/Rho kinase pathway and improve cancer therapy.

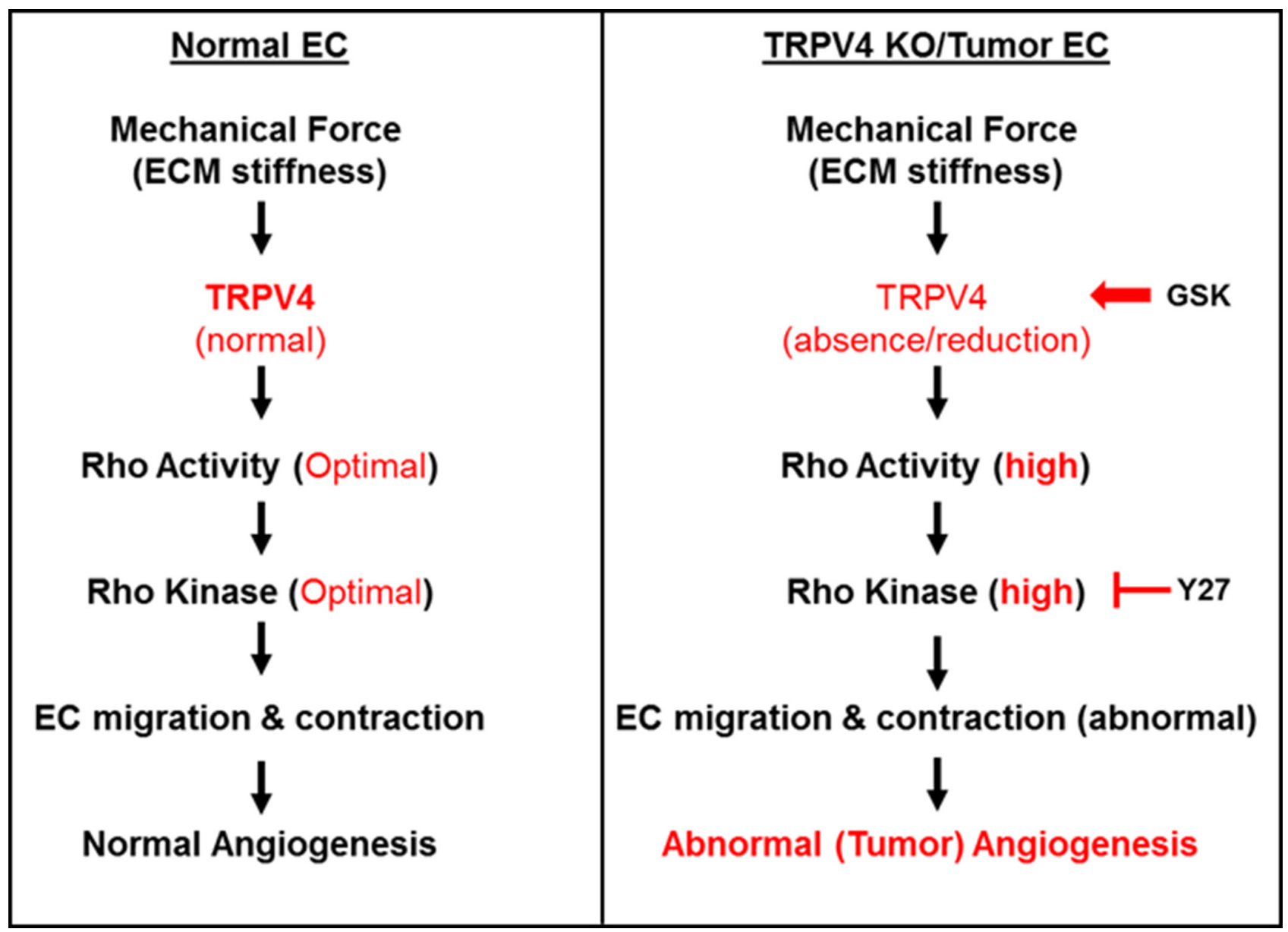

Figure 5: TRPV4-dependent mechanotransduction during angiogenesis. A schematic representation of TRPV4-mediated mechanical signaling in normal and TRPV4KO/TRPV4-deficient (tumor) endothelial cells. In normal endothelial cells, TRPV4 senses mechanical force (ECM stiffness) and induces optimal Rho/Rho kinase activation necessary for endothelial migration and contraction which is required for partial cell rounding and angiogenesis. However, absence (TRPV4KO EC) or reduction (Tumor EC) in TRPV4 expression and function results in high basal Rho/Rho kinase activation, leading to abnormal (tumor) angiogenesis. This abnormal tumor vasculature can be normalized by restoring mechanosensitivity through pharmacological activation of TRPV4 in tumor endothelial cells (TRPV4-deficient) with GSK1016790A or inhibition of Rho kinase in TRPV4KO EC with Y-27632. Overall, these findings suggest that targeting TRPV4/Rho kinase-mediated mechanotransduction may be a novel therapy for tumor vascular normalization and improving anticancer drug delivery. 


\section{METHODS}

\section{Cell culture}

Cells from WT-C57BL/6 (WT) and TRPV4KO mice were isolated using the aortic explant method, as described previously [64-65]. Aortic rings were carefully removed from Matrigel after 7 days and EC were isolated, washed, and plated on $0.1 \%$ gelatin coated dishes. The isolated primary $\mathrm{EC}$ were cultured in a defined media, as previously described $[12,64]$, in a $37^{\circ} \mathrm{C}, 5 \% \mathrm{CO}_{2}$ incubator, split at $\sim 90-95 \%$ confluence, and used between passages 1-8. Cells were characterized for the presence of endothelial cell marker (CD31) and the absence of alpha-smooth muscle actin via Western blot, $\mathrm{qPCR}$, and immunocytochemistry. Normal endothelial cells (NEC) were characterized and cultured as described previously $[12,33,64,66]$ and mouse Lewis Lung Carcinoma (LLC) cells were cultured in high glucose DMEM medium supplemented with $10 \%$ FBS and antibiotic/mycotic mix at $37^{\circ} \mathrm{C}$.

\section{Aortic ring assay}

Isolated aortic rings from WT and TRPV4KO mice, as previously described [64, 65], were carefully placed between 2 layers of Matrigel (BD Biosciences) and supplemented with defined endothelial cell medium. Aortic explants were monitored and imaged from Day 0 to Day 5 for vessel sprouting. The average number of sprouting vessels was calculated for Day 5 and data are expressed as fold increased from WT explants.

\section{Matrigel plug assay}

WT and TRPV4KO mice were subcutaneously injected at the flank on both sides with growth factor-reduced Matrigel (Becton Dickinson), supplemented with basic fibroblast growth factor (bFGF; R\&D Biosystems) (125 ng/ plug), heparin sulfate (Sigma Aldrich) $(15 \mu \mathrm{g} / \mathrm{plug})$, and vascular endothelial growth factor (VEGF; R\&D Systems) (1.2 ng/plug). Plugs were excised 2 weeks post implantation, fixed in 4\% PFA, and sectioned for immunohistochemical analysis.

\section{BrdU proliferation assay}

Cells were plated equally at low density on cover glasses for 24 hours, prior to treatment with $10 \mu \mathrm{M}$ BrdU (Abcam) for 2 hours. Cells were then fixed in 4\% PFA and stained as previously described [50]. Images were captured using an Olympus IX71-fluorcescence microscope and analyzed with ImageJ software (NIH). Quantitative analysis was performed by measuring BrdU positive cells in WT and TRPV4KO EC ((number of BrdU positive cells/total number of cells)*100).

\section{qPCR}

RNA was isolated from WT and TRPV4KO EC using the RNeasy Mini Kit (Qiagen) and measured with the NanoDrop 2000 UV-Vis Spectrometer. cDNA was synthesized with qscript cDNA SuperMix (Quanta Biosciences) and the Fast SYBR green master mix (Applied Biosystems) was used for qPCR analysis on the Fast Real-Time PCR system (Applied Biosystems). The following real time primers were obtained from IDT technologies: CD31 and GAPDH. Gene expression was made relative to GAPDH and $\Delta \triangle \mathrm{CT}$ values were expressed as fold change over WT.

\section{Western blot}

Cells were lysed in Triton X-100, containing protease and phosphatase inhibitor cocktails (Boston Bioproducts). Lysates were loaded onto $8 \%$ or $10 \%$ acrylamide gels for separation via electrophoresis and transferred onto a PVDF membrane. The membrane was blocked in 5\% milk in TBS with $0.1 \%$ Tween-20 (TBST), followed by overnight incubation with primary antibodies: anti-TRPV4 (1:300; Alomone Labs), tubulin (1:5000; Abcam), anti-phospho-ERK1/2 (1:1000; Cell Signaling), and total ERK1/2 (1:1000; Cell Signaling). After washing with TBST, membranes were incubated with the appropriate secondary antibodies, goat anti-rabbit $(1: 20,000)$ or goat anti-mouse $(1: 20,000)$, conjugated with horseradish peroxidase (Jackson Labs). Signals were detected with chemiluminescent substrates (Thermo Scientific) and developed with a FluorChem M Simple Imager (Protein Simple).

\section{Migration assay}

Cells were plated on 6 well-plates and grown to $95 \%$ confluency. Cells were washed with PBS or serum free media to remove traces of growth factors. The scratch was made using a $200 \mu \mathrm{L}$ tip and images were captured at 0 hour and 12 hours using an Olympus IX-51 brightfield microscope. Percent migration was quantified with Image J software (NIH) using the formula ((area of scratch a $0 \mathrm{hr}$ - area of scratch at $12 \mathrm{hr}$ )/area of scratch at $\left.(\mathrm{hr})^{*} 100\right)$ ).

\section{D angiogenesis assay}

Cells $\left(8 \times 10^{4}\right.$ cells/well $)$ were plated on growth factor-reduced Matrigel (BD Biosciences) in a 48 wellplate and kept at $37^{\circ} \mathrm{C}$ for up to 8 hours $[12,33]$. For assays performed in the presence of Rho kinase (ROCK) inhibitor, cells were treated with Y-27632 $(10 \mu \mathrm{M})$ prior to plating. Images were obtained with an Olympus IX-51 brightfield microscope and tube length was measured using Image J software (NIH). 


\section{Rho activation assay}

The Rhotekin-RBD affinity precipitation assay was used to analyze Rho activity, as previously described $[12,33,67]$. Briefly, cell lysates were incubated with GSTRhotekin-RBD beads (Cytoskeleton Inc) for 1 hour at $4^{\circ} \mathrm{C}$, followed by centrifugation and washing of the beads with wash buffer. Bound GTP-Rho was extracted with SDS sample buffer, probed for RhoA mAB (Santa Cruz) via Western blot, and GTP-Rho activity was analyzed from the resulting densitometric data. Active RhoA levels were normalized to total RhoA protein and values were expressed as fold change over WT.

\section{Tumor model}

All animal experiments were performed according to an approved protocol by Northeast Ohio Medical University, IACUC. Mouse Lewis Lung Carcinoma (LLC) cells $\left(2 \times 10^{6}\right)$ were injected into the flank on both sides of TRPV4KO mice [12]. Once tumors became palpable (after 7 days), six to eight mice/group were used for the following four treatment groups: (1) Control (2) Rho kinase inhibitor, Y-27632 (3) Cisplatin (4) Y-27632 + Cisplatin. Mice were given daily i.p. injections of Y-27632 (10 mg/ $\mathrm{kg}$ ) in groups 2 and 4. Anti-cancer drug, Cisplatin ( $3 \mathrm{mg} /$ $\mathrm{kg}$ ) was given once a week to group 3, and in group 4, Cisplatin was given once/week, 2-4 days post-treatment with Y-27632. Treatments were administered from Day 7 to Day 21, with the control group receiving saline as a vehicle. Calipers were used to measure tumor size at Day 7 , Day 14, and Day 21 and tumor volumes were calculated from the formula $\left(4 / 3 * \mathrm{pi}^{*}\right.$ length* $\left.\left((\text { width } / 2)^{2}\right)\right)$. Mice were sacrificed at Day 21 and tumor tissues were collected for immunohistochemical analysis.

\section{Immunohistochemistry}

Matrigel plug or tumor tissue sections, cut at approximately $10 \mu \mathrm{M}$ thickness, were fixed and permeabilized in ice cold acetone (20 min), washed with Tris buffered saline (TBS), and incubated overnight with anti-CD31 (1:50; Invitrogen) to visualize vessels. Tumor tissues were also stained for $\alpha$-SMA (1:200; Sigma Aldrich) to visualize pericytes. Sections were washed, and incubated with the appropriate secondary antibody tagged with Alexa Fluor-488 or Alexa Fluor-594 (Invitrogen) and mounted with DAPI (Vector Labs). Images were captured with an Olympus IX-81 microscope and quantified with Image J software (NIH). Percent pericyte coverage was analyzed by dividing the number of $\mathrm{CD} 31^{+} / \alpha-\mathrm{SMA}^{+}$cells by the total number of $\mathrm{CD} 31^{+}$cells multiplied by 100 .

\section{Immunocytochemistry}

WT and TRPV4KO EC were fixed in 4\% paraformaldehyde (20-30 min), washed with PBS, and permeabilized with $0.25 \%$ Triton X-100 (15 min), followed by blocking with serum containing medium for 30-60 min at room temperature. Cells were then incubated with $\alpha$-SMA (1:200; Sigma Aldrich) for 1 hour at room temperature or overnight at $4^{\circ} \mathrm{C}$. Following washing, cells were incubated with Alexa Fluor-488 conjugated secondary antibody (1:500: Invitrogen) and mounted with DAPI (Vector Labs). Images were captured using an Olympus IX-71 fluorescence microscope. Lung fibroblasts were used as a positive control.

\section{Cell spreading on varying stiffness ECM substrates}

Transglutaminase-crosslinked gelatin hydrogels of increasing stiffness were prepared using 3\%, 5\%, and $10 \%(\mathrm{w} / \mathrm{v})$ final gelatin concentration, corresponding to 98,370 , and $2280 \mathrm{~Pa}$, and incubated at $4^{\circ} \mathrm{C}$ overnight to stabilize crosslinking $[12,33]$. Cells in regular culture medium were plated at low density (to minimize cell-cell interactions) and allowed to spread for $6 \mathrm{~h}$. Images were captured using an Olympus IX81 microscope and cell area was calculated using ImageJ software (NIH).

\section{GRANT SUPPORT}

This work was supported by American Heart Association (AHA) Grant-in-Aid (14GRNT20380935), National Institutes of Health (NIH) (R01HL119705 and R15CA202847), and start-up funds from NEOMED (CKT).

\section{CONFLICTS OF INTEREST}

\section{None.}

\section{Authors' contributions}

R.T., H.C., and R.A. performed research, analyzed the data, and edited the manuscript. S.P. and A.K. edited the manuscript. C.K.T. designed, interpreted and analyzed data as well as wrote the manuscript.

\section{REFERENCES}

1. Hanahan D, Weinberg RA. Hallmarks of cancer: the next generation. Cell. 2011; 144:646-674.

2. Carmeliet P. Angiogenesis in health and disease. Nat Med. 2003; 9:653-660.

3. Carmeliet P, Jain RK. Molecular mechanisms and clinical applications of angiogenesis. Nature. 2011; 473:298-307.

4. Fukumura D, Duda DG, Munn LL, Jain RK. Tumor microvasculature and microenvironment: novel insights through intravital imaging in pre-clinical models. Microcirculation. 2010; 17:206-225.

5. Bergers G, Hanahan D. Modes of resistance to antiangiogenic therapy. Nature reviews Cancer. 2008; 8:592-603. 
6. Casanovas O, Hicklin DJ, Bergers G, Hanahan D. Drug resistance by evasion of antiangiogenic targeting of VEGF signaling in late-stage pancreatic islet tumors. Cancer cell. 2005; 8:299-309.

7. Fiorio Pla A, Gkika D. Emerging role of TRP channels in cell migration: from tumor vascularization to metastasis. Front Physiol. 2013; 4:311.

8. Lehen'kyi V, Prevarskaya N. (2011). Study of TRP Channels in Cancer Cells. In: Zhu MX, ed. TRP Channels. (Boca Raton (FL).

9. Lehen'kyi V, Prevarskaya N. Oncogenic TRP channels. Advances in experimental medicine and biology. 2011; 704:929-945.

10. Prevarskaya N, Skryma R, Shuba Y. Calcium in tumour metastasis: new roles for known actors. Nature reviews Cancer. 2011; 11:609-618.

11. Shapovalov G, Lehen'kyi V, Skryma R, Prevarskaya N. TRP channels in cell survival and cell death in normal and transformed cells. Cell calcium. 2011; 50:295-302.

12. Adapala RK, Thoppil RJ, Ghosh K, Cappelli HC, Dudley AC, Paruchuri S, Keshamouni V, Klagsbrun M, Meszaros JG, Chilian WM, Ingber DE, Thodeti CK. Activation of mechanosensitive ion channel TRPV4 normalizes tumor vasculature and improves cancer therapy. Oncogene. 2015.

13. Earley S, Pauyo T, Drapp R, Tavares MJ, Liedtke W, Brayden JE. TRPV4-dependent dilation of peripheral resistance arteries influences arterial pressure. Am J Physiol Heart Circ Physiol. 2009; 297:H1096-1102.

14. Hartmannsgruber V, Heyken WT, Kacik M, Kaistha A, Grgic I, Harteneck C, Liedtke W, Hoyer J, Kohler R. Arterial response to shear stress critically depends on endothelial TRPV4 expression. PloS one. 2007; 2:e827.

15. Kohler R, Heyken WT, Heinau P, Schubert R, Si H, Kacik M, Busch C, Grgic I, Maier T, Hoyer J. Evidence for a functional role of endothelial transient receptor potential V4 in shear stress-induced vasodilatation. Arterioscler Thromb Vasc Biol. 2006; 26:1495-1502.

16. Liedtke W. TRPV channels' role in osmotransduction and mechanotransduction. Handb Exp Pharmacol. 2007; 473-487.

17. Matthews BD, Thodeti CK, Tytell JD, Mammoto A, Overby DR, Ingber DE. Ultra-rapid activation of TRPV4 ion channels by mechanical forces applied to cell surface beta1 integrins. Integr Biol (Camb). 2010; 2:435-442.

18. Mendoza SA, Fang J, Gutterman DD, Wilcox DA, Bubolz AH, Li R, Suzuki M, Zhang DX. TRPV4-mediated endothelial $\mathrm{Ca} 2+$ influx and vasodilation in response to shear stress. Am J Physiol Heart Circ Physiol. 2010; 298:H466-476.

19. Thodeti CK, Matthews B, Ravi A, Mammoto A, Ghosh K, Bracha AL, Ingber DE. TRPV4 channels mediate cyclic strain-induced endothelial cell reorientation through integrin-to-integrin signaling. Circulation research. 2009; 104:1123-1130.
20. Rath N, Olson MF. Rho-associated kinases in tumorigenesis: re-considering ROCK inhibition for cancer therapy. EMBO reports. 2012; 13:900-908.

21. Nakabayashi H, Shimizu K. HA1077, a Rho kinase inhibitor, suppresses glioma-induced angiogenesis by targeting the Rho-ROCK and the mitogen-activated protein kinase kinase/extracellular signal-regulated kinase (MEK/ ERK) signal pathways. Cancer science. 2011; 102:393-399.

22. Nakajima M, Hayashi K, Katayama K, Amano Y, Egi Y, Uehata M, Goto N, Kondo T. Wf-536 prevents tumor metastasis by inhibiting both tumor motility and angiogenic actions. European journal of pharmacology. 2003; 459:113-120.

23. Ogata S, Morishige K, Sawada K, Hashimoto K, Mabuchi S, Kawase C, Ooyagi C, Sakata M, Kimura T. Fasudil inhibits lysophosphatidic acid-induced invasiveness of human ovarian cancer cells. International journal of gynecological cancer. 2009; 19:1473-1480.

24. Patel RA, Liu Y, Wang B, Li R, Sebti SM. Identification of novel ROCK inhibitors with anti-migratory and antiinvasive activities. Oncogene. 2014; 33:550-555.

25. Somlyo AV, Bradshaw D, Ramos S, Murphy C, Myers CE, Somlyo AP. Rho-kinase inhibitor retards migration and in vivo dissemination of human prostate cancer cells. Biochemical and biophysical research communications. 2000; 269:652-659.

26. Teiti I, Florie B, Pich C, Gence R, Lajoie-Mazenc I, Rochaix P, Favre G, Tilkin-Mariame AF. In vivo Effects in Melanoma of ROCK Inhibition-Induced FasL Overexpression. Frontiers in oncology. 2015; 5:156.

27. Bryan BA, D'Amore PA. What tangled webs they weave: Rho-GTPase control of angiogenesis. Cellular and molecular life sciences. 2007; 64:2053-2065.

28. Yin L, Morishige K, Takahashi T, Hashimoto K, Ogata S, Tsutsumi S, Takata K, Ohta T, Kawagoe J, Takahashi K, Kurachi H. Fasudil inhibits vascular endothelial growth factor-induced angiogenesis in vitro and in vivo. Molecular cancer therapeutics. 2007; 6:1517-1525.

29. Wermke M, Camgoz A, Paszkowski-Rogacz M, Thieme S, von Bonin M, Dahl A, Platzbecker U, Theis M, Ehninger G, Brenner S, Bornhauser M, Buchholz F. RNAi profiling of primary human AML cells identifies ROCK1 as a therapeutic target and nominates fasudil as an antileukemic drug. Blood. 2015; 125:3760-3768.

30. Ying H, Biroc SL, Li WW, Alicke B, Xuan JA, Pagila R, Ohashi Y, Okada T, Kamata Y, Dinter H. The Rho kinase inhibitor fasudil inhibits tumor progression in human and rat tumor models. Molecular cancer therapeutics. 2006; 5:2158-2164.

31. Ingber DE. Extracellular matrix as a solid-state regulator in angiogenesis: identification of new targets for anti-cancer therapy. Semin Cancer Biol. 1992; 3:57-63.

32. Ingber DE. Mechanical signaling and the cellular response to extracellular matrix in angiogenesis and cardiovascular physiology. Circulation research. 2002; 91:877-887. 
33. Ghosh K, Thodeti CK, Dudley AC, Mammoto A, Klagsbrun M, Ingber DE. Tumor-derived endothelial cells exhibit aberrant Rho-mediated mechanosensing and abnormal angiogenesis in vitro. Proc Natl Acad Sci U S A. 2008; 105:11305-11310.

34. Paszek MJ, Weaver VM. The tension mounts: mechanics meets morphogenesis and malignancy. Journal of mammary gland biology and neoplasia. 2004; 9:325-342.

35. Paszek MJ, Zahir N, Johnson KR, Lakins JN, Rozenberg GI, Gefen A, Reinhart-King CA, Margulies SS, Dembo M, Boettiger D, Hammer DA, Weaver VM. Tensional homeostasis and the malignant phenotype. Cancer cell. 2005; 8:241-254.

36. Duda DG, Batchelor TT, Willett CG, Jain RK. VEGFtargeted cancer therapy strategies: current progress, hurdles and future prospects. Trends Mol Med. 2007; 13:223-230.

37. Ferrara N. VEGF as a therapeutic target in cancer. Oncology. 2005; 69:11-16.

38. Munaron L. Intracellular calcium, endothelial cells and angiogenesis. Recent patents on anti-cancer drug discovery. 2006; 1:105-119.

39. Munaron L, Fiorio Pla A. Endothelial calcium machinery and angiogenesis: understanding physiology to interfere with pathology. Current medicinal chemistry. 2009; 16:4691-4703.

40. Munaron L, Genova T, Avanzato D, Antoniotti S, Fiorio Pla A. Targeting calcium channels to block tumor vascularization. Recent patents on anti-cancer drug discovery. 2013; 8:27-37.

41. Park YR, Chun JN, So I, Kim HJ, Baek S, Jeon JH, Shin SY. Data-driven Analysis of TRP Channels in Cancer: Linking Variation in Gene Expression to Clinical Significance. Cancer genomics \& proteomics. 2016; 13:83-90.

42. Hamdollah Zadeh MA, Glass CA, Magnussen A, Hancox JC, Bates DO. VEGF-mediated elevated intracellular calcium and angiogenesis in human microvascular endothelial cells in vitro are inhibited by dominant negative TRPC6. Microcirculation. 2008; 15:605-614.

43. Jho D, Mehta D, Ahmmed G, Gao XP, Tiruppathi C, Broman M, Malik AB. Angiopoietin-1 opposes VEGFinduced increase in endothelial permeability by inhibiting TRPC1-dependent Ca2 influx. Circulation research. 2005; 96:1282-1290.

44. Yu PC, Gu SY, Bu JW, Du JL. TRPC1 is essential for in vivo angiogenesis in zebrafish. Circulation research. 2010; 106:1221-1232.

45. Ge R, Tai Y, Sun Y, Zhou K, Yang S, Cheng T, Zou Q, Shen F, Wang Y. Critical role of TRPC6 channels in VEGFmediated angiogenesis. Cancer letters. 2009; 283:43-51.

46. Troidl C, Troidl K, Schierling W, Cai WJ, Nef H, Mollmann H, Kostin S, Schimanski S, Hammer L, Elsasser A, Schmitz-Rixen T, Schaper W. Trpv4 induces collateral vessel growth during regeneration of the arterial circulation. J Cell Mol Med. 2009; 13:2613-2621.
47. Kohler R, Hoyer J. Role of TRPV4 in the Mechanotransduction of Shear Stress in Endothelial Cells. In: Liedtke WB, Heller S, eds. TRP Ion Channel Function in Sensory Transduction and Cellular Signaling Cascades. (Boca Raton (FL). 2007.

48. Gradilone SA, Masyuk TV, Huang BQ, Banales JM, Lehmann GL, Radtke BN, Stroope A, Masyuk AI, Splinter PL, LaRusso NF. Activation of Trpv4 reduces the hyperproliferative phenotype of cystic cholangiocytes from an animal model of ARPKD. Gastroenterology. 2010; 139:304-314 e302.

49. Zaika O, Mamenko M, Berrout J, Boukelmoune N, O'Neil RG, Pochynyuk O. TRPV4 dysfunction promotes renal cystogenesis in autosomal recessive polycystic kidney disease. J Am Soc Nephrol. 2013; 24:604-616.

50. Thoppil RJ, Adapala RK, Cappelli HC, Kondeti V, Dudley AC, Gary Meszaros J, Paruchuri S, Thodeti CK. TRPV4 channel activation selectively inhibits tumor endothelial cell proliferation. Scientific reports. 2015; $5: 14257$.

51. Kusuyama J, Bandow K, Shamoto M, Kakimoto K, Ohnishi T, Matsuguchi T. Low intensity pulsed ultrasound (LIPUS) influences the multilineage differentiation of mesenchymal stem and progenitor cell lines through ROCK-Cot/Tpl2-MEK-ERK signaling pathway. The Journal of biological chemistry. 2014; 289:10330-10344.

52. Ohashi K, Nagata K, Maekawa M, Ishizaki T, Narumiya S, Mizuno K. Rho-associated kinase ROCK activates LIMkinase 1 by phosphorylation at threonine 508 within the activation loop. The Journal of biological chemistry. 2000; 275:3577-3582.

53. Samarakoon R, Chitnis SS, Higgins SP, Higgins CE, Krepinsky JC, Higgins PJ. Redox-induced Src kinase and caveolin-1 signaling in TGF-beta1-initiated SMAD2/3 activation and PAI-1 expression. PloS one. 2011; 6:e22896.

54. Sanz-Moreno V, Gaggioli C, Yeo M, Albrengues J, Wallberg F, Viros A, Hooper S, Mitter R, Feral CC, Cook M, Larkin J, Marais R, Meneguzzi G, et al. ROCK, JAK1 signaling cooperate to control actomyosin contractility in tumor cells and stroma. Cancer cell. 2011; 20:229-245.

55. Keely PJ. Mechanisms by which the extracellular matrix and integrin signaling act to regulate the switch between tumor suppression and tumor promotion. Journal of mammary gland biology and neoplasia. 2011; 16:205-219.

56. Ader I, Delmas C, Bonnet J, Rochaix P, Favre G, Toulas C, Cohen-Jonathan-Moyal E. Inhibition of Rho pathways induces radiosensitization and oxygenation in human glioblastoma xenografts. Oncogene. 2003; 22:8861-8869.

57. Deng L, Li G, Li R, Liu Q, He Q, Zhang J. Rho-kinase inhibitor, fasudil, suppresses glioblastoma cell line progression in vitro and in vivo. Cancer biology \& therapy. 2010; 9:875-884.

58. Xue F, Takahara T, Yata Y, Xia Q, Nonome K, Shinno E, Kanayama M, Takahara S, Sugiyama T. Blockade of Rho/ Rho-associated coiled coil-forming kinase signaling can 
prevent progression of hepatocellular carcinoma in matrix metalloproteinase-dependent manner. Hepatology research. 2008; 38:810-817.

59. Davies PF, Spaan JA, Krams R. Shear stress biology of the endothelium. Ann Biomed Eng. 2005; 33:1714-1718.

60. Mammoto A, Connor KM, Mammoto T, Yung CW, Huh D, Aderman CM, Mostoslavsky G, Smith LE, Ingber DE. A mechanosensitive transcriptional mechanism that controls angiogenesis. Nature. 2009; 457:1103-1108.

61. Mammoto A, Mammoto T, Ingber DE. Rho signaling and mechanical control of vascular development. Curr Opin Hematol. 2008; 15:228-234.

62. Bryan BA, Dennstedt E, Mitchell DC, Walshe TE, Noma K, Loureiro R, Saint-Geniez M, Campaigniac JP, Liao JK, D'Amore PA. RhoA/ROCK signaling is essential for multiple aspects of VEGF-mediated angiogenesis. FASEB J. 2010; 24:3186-3195.

63. Van der Veldt AA, Lubberink M, Bahce I, Walraven M, de Boer MP, Greuter HN, Hendrikse NH, Eriksson J, Windhorst AD, Postmus PE, Verheul HM, Serne EH,
Lammertsma AA, et al. Rapid decrease in delivery of chemotherapy to tumors after anti-VEGF therapy: implications for scheduling of anti-angiogenic drugs. Cancer cell. 2012; 21:82-91.

64. Adapala RK, Talasila PK, Bratz IN, Zhang DX, Suzuki M, Meszaros JG, Thodeti CK. PKCalpha mediates acetylcholine-induced activation of TRPV4-dependent calcium influx in endothelial cells. Am J Physiol Heart Circ Physiol. 2011; 301:H757-765.

65. Baker M, Robinson SD, Lechertier T, Barber PR, Tavora B, D'Amico G, Jones DT, Vojnovic B, Hodivala-Dilke K. Use of the mouse aortic ring assay to study angiogenesis. Nature protocols. 2012; 7:89-104.

66. Dudley AC, Khan ZA, Shih SC, Kang SY, Zwaans BM, Bischoff J, Klagsbrun M. Calcification of multipotent prostate tumor endothelium. Cancer cell. 2008; 14:201-211.

67. Thodeti CK, Massoumi R, Bindslev L, Sjolander A. Leukotriene D4 induces association of active RhoA with phospholipase C-gammal in intestinal epithelial cells. Biochem J. 2002; 365:157-163. 